\title{
AA2219 Aluminum Alloy Processed via Multi-Axial Forging in Cryogenic and Ambient Environments
}

\author{
Amin Azimi ${ }^{1}$, Gbadebo Moses Owolabi ${ }^{1}$, Hamid Fallahdoost ${ }^{2}$, Nikhil Kumar ${ }^{1}$, Horace Whitworth ${ }^{1}$, \\ \& Grant Warner ${ }^{1}$ \\ ${ }^{1}$ Department of Mechanical Engineering, Howard University, Washington, DC, USA \\ ${ }^{2}$ Department of Mechanical Engineering, Materials Science and Engineering Program, Binghamton University \\ (SUNY), Binghamton, NY, USA \\ Correspondence: Amin Azimi, Department of Mechanical Engineering, Howard University, Washington, DC, \\ USA. Tel: 1202-806-6594. E-mail: amin.azimi@bison.howard.edu; aminazimi.kntu@gmail.com
}

Received: January 10, 2019

doi: 10.5539/jmsr.v8n2p1
Accepted: February 12, 2019

URL: https://doi.org/10.5539/jmsr.v8n2p1
Online Published: March 6, 2019

\begin{abstract}
This paper presents the microstructure and the mechanical behavior of nanocrystalline AA2219 processed by multi axial forging (MAF) at ambient and cryogenic temperatures. The X-ray diffraction pattern and transmission electron microscopy micrographs in the initial microstructure characterization indicate a more effective severe plastic deformation during the cryogenic MAF than the same process conducted at room temperature. MAF at cryogenic temperature results in crystallite size reduction to nanoscales as well as second phase particles breakage to finer particles which are the crucial factors to increasing the mechanical properties of the material. Fractography analysis and tensile tests results show that cryogenic forging does not only increase the mechanical strength and toughness of the alloys significantly, but also improves the ductility of the material in comparison with the conventional forging. In this comparative regard, cryogenic processing provides $44 \%$ increase in the tensile strength of the material only after 2 forging cycles when compared to the room temperature process. In addition, further forging process to the next cycles slightly enhances the tensile strength at the expense of ductility due to less ability of the dislocations to accumulate. However, the ductility of the ambient temperature forged samples decreases at a faster rate than that of cryoforged samples.
\end{abstract}

Keywords: Ultrafine Grained AA2219, Cryogenic Forging, Quasi-Static Mechanical Behavior, Microstructure Investigation, Fractography Analysis

\section{Introduction}

AA2219 is a heat-treatable material based on aluminum-copper system with metastable $\mathrm{AlCu}\left(\theta^{\prime}, \theta^{\prime \prime}\right)$ as the major strengthening second phase (Elgallad, Zhang, \& Chen, 2015). High strength-to-weight ratio, excellent weldability, machinability and superior cryogenic properties lead to the alloy being increasingly used as an engineering material in the aircraft and the automotive sectors as a structural material (Asm, 1990; Owolabi et al., 2017). It is widely accepted that severe plastic deformation (SPD) process on metals offers grain refinement and formation of ultrafine-grained (UFG) microstructure with a mean grain size in the submicron/nano meter range (Nejadseyfi, Shokuhfar, Dabiri, \& Azimi, 2015; Valiev, Islamgaliev, \& Alexandrov, 2000). UFG materials are structurally distinguished with a significant density of grain boundaries, which may substantially modify their physical and mechanical behavior in comparison to the equivalent coarse-grained counterpart (Nejadseyfi, Shokuhfar, Azimi, \& Shamsborhan, 2015; Xu, Zhao, Wu, Ma, \& Guan, 2007). The mechanical properties of metals and alloys could potentially be enhanced via this grain size reduction which has been adequately discussed by the Hall-Petch equation (Hansen, 2004).

One of the most promising SPD methods is Multi Axial Forging (MAF), which is a relatively uncomplicated method to attain UFG materials (Rao, Singh, \& Jayaganthan, 2014). MAF involves repeated compression on three orthogonal directions conducted with a change in the axis by $90^{\circ}$ in every pass which helps in achieving a uniform texture with low aspect ratio grains structure. MAF does not only have the potential for producing large processed samples which is desired for industrial applications, but it also keeps the original configuration with least distortion even after many successive cycles which provides significant point of interest among metals forming researchers (Cherukuri \& Srinivasan, 2006; Sitdikov, Sakai, Goloborodko, \& Miura, 2004). However, 
SPD at room temperature cannot be very effective to form UFG materials due to recrystallization and dynamic recovery of grains during the plastic deformation (Kumar Singh, Ghosh, \& Mula, 2016). As a novel approach, dynamic recovery is suppressed at liquid nitrogen temperatures and accordingly facilitates the formation of UFG structures (Magalhães, Kliauga, Ferrante, \& Sordi, 2017; Panigrahi, Jayaganthan, \& Chawla, 2008).

It is thoroughly established that UFG microstructures deformed by SPD exhibit different mechanical behavior when compare with large grained materials, such as increased strength associated with reduced elongation and yield drop due to the unexpected beginning of necking (Hung, Sun, Yu, Kao, \& Chang, 2005). The particular mechanical characteristics in these materials such as high ultimate strength, different ductility and plastic deformation profile reveal an alteration in their deformation behavior. Intragrain dislocation slip and twinning govern the plastic deformation of the metals and alloys with a coarse grain structure (Ivanov \& Naydenkin, 2012). However, diffusion-controlled mechanisms such as grain boundary sliding and grain rotation can be efficiently activated in UFG materials, since the activation energy of the plastic deformation is roughly equal to the activation energy of the grain boundary diffusion (Ivanov \& Naydenkin, 2012). This might be attributed to the high volume fraction of the grain boundaries in non-equilibrium state.

To the best of the authors' knowledge, no literature is available on the quasi-static mechanical behavior of the UFG AA2219 processed by cryogenic forging. Thus, the current research is to provide insight into the microstructure and mechanical response of the cryogenic processed samples by tensile strength and Vickers indentation tests. The 2, 4 and 6 MAF cycles are evaluated and sample with same MAF procedure at room temperature is considered as a reference. Moreover, the microstructure fractured surfaces of the tested samples were studied to realize failure mechanisms in such UFG materials.

\section{Experimental Procedure}

AA2219 alloy (with the chemical composition summarized in Table 1) is used in the present research. Initially, the specimens were placed in argon atmosphere furnace with $500^{\circ} \mathrm{C}$ temperature to be solution treated for a period of 10 hours. This treatment was completed by quenching samples in water at ambient temperature. The MAF process was conducted using screw forging apparatus at a strain rate of 10s-1. True strain of $(\Delta \varepsilon)=\ln (1 / 1.18)=-0.165$ was successively applied by axial compression for one forging pass. Cumulatively, the strain in one cycle was determined as $\left(\sum \varepsilon_{\mathrm{n}=1}\right)=\left|\Delta \varepsilon_{1}+\Delta \varepsilon_{2}+\Delta \varepsilon_{3}\right|=-0.495$. The initial forging axis was aligned with the extrusion direction of the reference specimen. To reach the cryogenic temperature, the samples were put inside liquid nitrogen for $15 \mathrm{~min}$. The solution treated materials were continually forged such that the forging axis was rotated by $90^{\circ}$ to retain the dimensional ratio of 1.18: 1.11: 1.0 after deformation completion. Prior to each pass, a 5-10 min quenching in liquid nitrogen was performed to keep the samples in thermal equilibrium with the liquid nitrogen. Eventually, the AA2219 materials were exposed to total strains of $-0.99,-1.98$ and -2.97 under 2 cycles ( 6 passes), 4 cycles (12 passes) and 6 cycles (18 passes), respectively.

The structural changes of the alloys as a result of the cryogenic forging were investigated by means of X-ray diffraction measurement (XRD). The XRD spectra were recorded at $2 \theta$ angle from $20^{\circ}$ to $80^{\circ}$ with a step size of $0.031^{\circ}$ utilizing a Philips vertical Scanning diffractometer at a voltage and electrical current of $40 \mathrm{kV}$ with $\mathrm{Cu}-\mathrm{K} \alpha$ radiation $(\lambda=0.15406 \mathrm{~nm})$. The subgrains structure and grain boundaries interaction in the UFG materials were studied with transmission electron microscopy (TEM; Model: FEI Technai) with a voltage of $200 \mathrm{kV}$. A scanning electron microscope (SEM) was employed to focus on the fractured surfaces and used to recognize the failure mechanisms in the deformed samples. The specimens were subjected to a microhardness test using a Vickers indenter at a load of $50 \mathrm{~g}$ and duration time of $10 \mathrm{~s}$. In addition, tensile tests to failure were conducted at a strain rate of $1 \mathrm{~mm} / \mathrm{min}$ by UTS-type machine (S-series H25k-S Model) at room temperature based on ASTM: E8M standard.

Table 1. Chemical composition of the AA2219 aluminum alloy

\begin{tabular}{lllllllllll}
\hline Elements & $\mathrm{Cu}$ & $\mathrm{Fe}$ & $\mathrm{Mg}$ & $\mathrm{Mn}$ & $\mathrm{Si}$ & $\mathrm{Ti}$ & $\mathrm{V}$ & $\mathrm{Zn}$ & $\mathrm{Zr}$ & $\mathrm{Al}$ \\
\hline $\mathrm{wt} \%$ & $5.8-6.8$ & 0.3 & 0.02 & $0.2-0.4$ & 0.2 & $0.02-0.1$ & $0.05-0.15$ & 0.1 & $0.1-0.25$ & $\mathrm{Bal}$. \\
\hline
\end{tabular}

\section{Results and Discussion}

\subsection{Microstructure Characterization}

Results of the XRD scattering pattern are represented in Figure 1 indicating the diffraction spectra of the room temperature forging (RTF) and cryoforging (CF) processed AA2219 alloy. The diffraction peaks of $\mathrm{Al}$ and $\mathrm{Al}_{2} \mathrm{Cu}$ 
confirm the existence of the $\mathrm{Al}_{2} \mathrm{Cu}$ second phase in the $\mathrm{Al}$ matrix. As a result of the cryogenic forging, broadening and intensity drop of the $\mathrm{Al}$ peaks are obvious. This can be an indication of the crystallite size refinement and an increase in the lattice strain of the CF alloys. The reduction of the dislocations mobility and allowing them to accumulate leads to the attainment of a higher steady state level in the materials processed with cryogenic SPD (Kumar Singh et al., 2016). The intense plastic strain induced at such low temperature leads to the formation and preservation of high density dislocations which facilitate the substructure consisting of ultra-fine grains. Tan et al. (Tan, Kibar, \& Gür, 2011) also reported a similar peak broadening and intensity reduction caused from the high dislocation densities and formation of UFG 6061 aluminum alloy during dissimilar channel angular pressing. In addition, $\mathrm{Al}_{2} \mathrm{Cu}$ phase in $\mathrm{RTF}$ samples appears with the stronger intensity than that of $\mathrm{CF}$ alloys. This can be attributed to a higher thermal energy and an increase of local temperature during the ambient forging process which provides the driving force for the reaction between $\mathrm{Al}$ and $\mathrm{Cu}$ to form $\mathrm{Al}_{2} \mathrm{Cu}$.

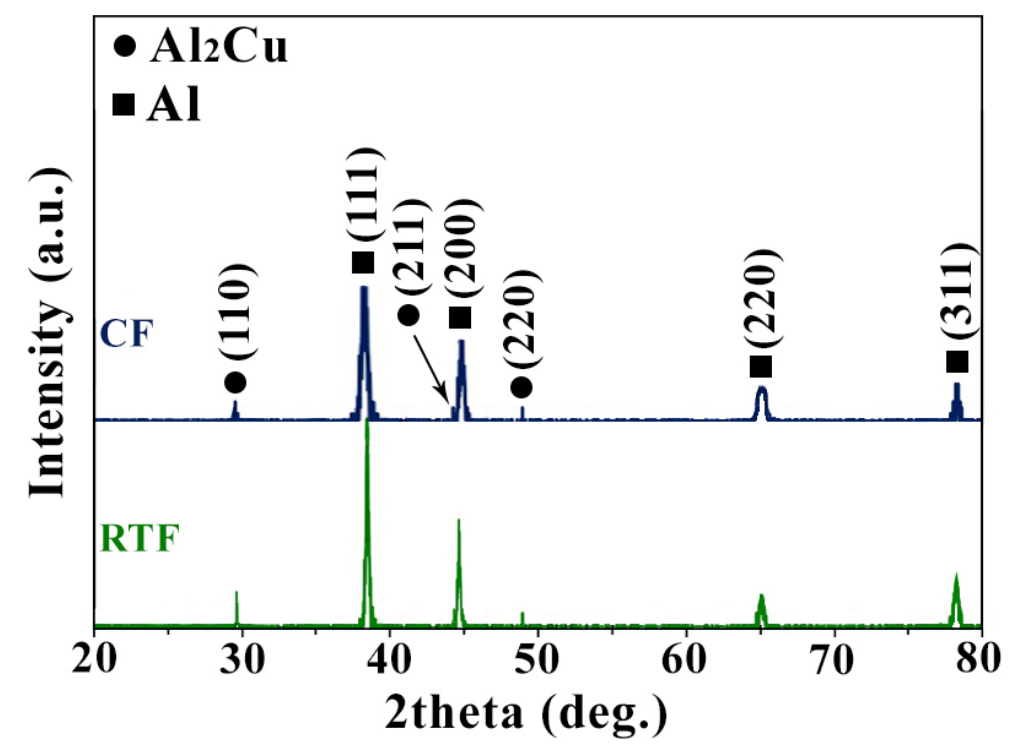

Figure 1. X-ray diffraction pattern of the RTF and CF processed AA2219

The TEM micrographs of the 6 cycles CF sample are illustrated in Figure 2 to reveal its subgrains structure. The microstructure of the CF sample exhibits the presence of heavily deformed grains associated with high density of dislocations. This can clearly be characterized from the magnified view of the TEM images as shown in Figure $2 \mathrm{~b}$.
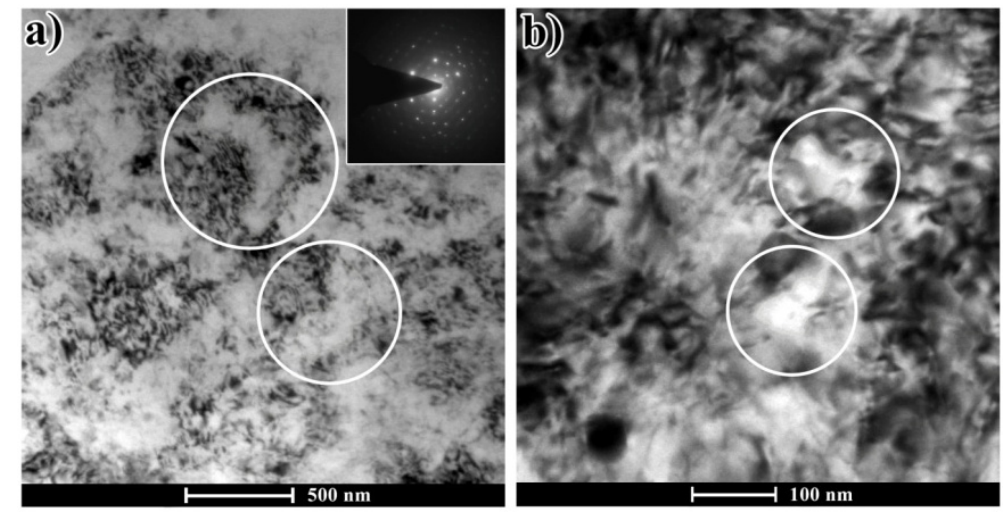

Figure 2. TEM micrographs of CF processed AA2219 in two different magnifications

Cell structures with grain boundaries (circle marks in Figure 2) are observable from this micrograph which lie in nano-metric scale. Furthermore, the continuous ring in the selected area electron diffraction (SAED) suggests the UFG structure in the TEM image. Cryogenic forging led to the formation of this distorted nanoscale subgrains and the development of nanoscale deformation twins at locally accumulated grain boundaries. This can be the 
result of the induced intense plastic deformation as well as suppression of the dynamic recovery and recrystallization mechanisms. These subgrains are mostly formed nearly equiaxed structure with dense dislocation walls. Chen et al. (Chen, Roven, Gireesh, Skaret, \& Hjelen, 2011) observed such high-density of dislocations and distorted grains with a substantial grain size refinement as a result of cryogenic equal-channel deformation of an Al-Mg alloy.

Figure 3 illustrates the schematics of microstructural modification mechanisms during the cryogenic and the ambient forgings of the AA2219 alloy in comparative view. With increasing plastic strain during the SPD process, more dislocations are activated and accumulated in the grain boundaries. A high elastic strain as a result atomic mismatch occurs in the accumulated dislocations areas. The generation of dislocation walls and dislocations tangles in the original grains leads to their successive transformation to sub-boundaries with small misorientation segregating single cells or subgrains (Azadmanjiri, Berndt, Kapoor, \& Wen, 2015). Subsequently, sub-boundaries develop to highly misoriented grain boundaries. The multi-directional deformation during the MAF may cause the alteration of the slip systems in the grain structure. As a result, the dislocations encounter with both dislocations in running slip systems and inactive dislocations created by the former MAF passes. Thus, the dense dislocation walls subdivided the original grains more effectively compared with the other conventional SPD techniques. However, forging at room temperature in a critical strain leads to a continuous recrystallization to minimize the free energy of the system. As soon as the rate of the dislocation proliferation is equilibrated with the rate of their annihilation, the increase in strain is no longer able to reach the subgrains size, which results in a stabilized grain size. On the contrary, forging at cryogenic temperature prevails over this destructive factor in grain refinement process by the suppression of the dynamic recovery of grains and grain boundaries structure. Figure 3 clearly exhibits this phenomenon and highlights the different grain refinement mechanisms between RTF and CF samples and the role of cryogenic temperature in the forging process.

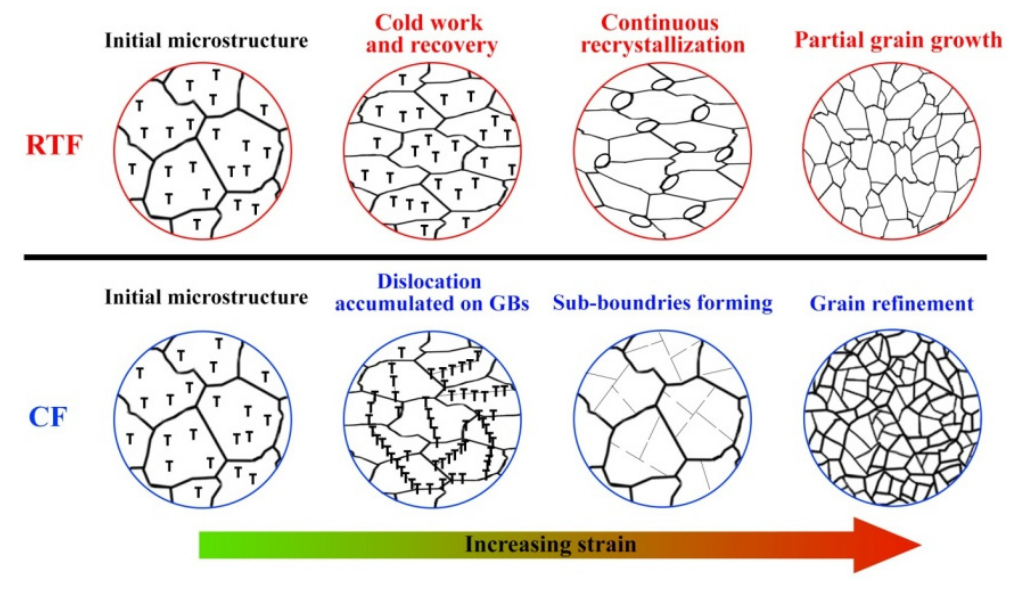

Figure 3. Schematics of microstructure modifications during cryogenic and ambient forgings

\subsection{Mechanical Behavior}

Figure 4 shows the stress-strain profiles for the quasi-static tensile tests conducted on the RTF and CF processed samples at 2, 4 and 6 forging cycles. Subsequently, the ultimate tensile strength (UTS) and the percentage elongation of the tested specimens are depicted in Figure 5. To provide insights into the effect of cryogenic forging on the properties of the material, the mechanical properties obtained from these curves such as the UTS, percentage ductility and toughness along with hardness measurements from the Vickers indentation test are quantitatively summarized in Table 2. As shown in Figure 4a for the RTF samples and Table 2, further forging process to the next cycles from 2 to 4 and then 6 cycles considerably improves the UTS of the RTF samples. $20 \%$ and $9 \%$ UTS enhancements were observed by increasing cycles from 2 to 4 and 4 to 6 , respectively. Crystallite size reduction causes by the imposed severe plastic deformation and work-hardening results in such mechanical properties enhancement. However, this process gradually continues at a decreasing rate due to thermomechanical equilibrium in kinetics of grains recrystallization and refinement. 
a)

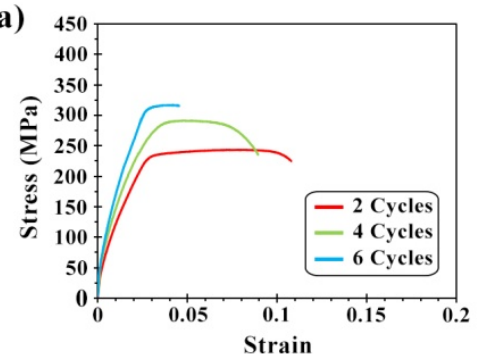

b)

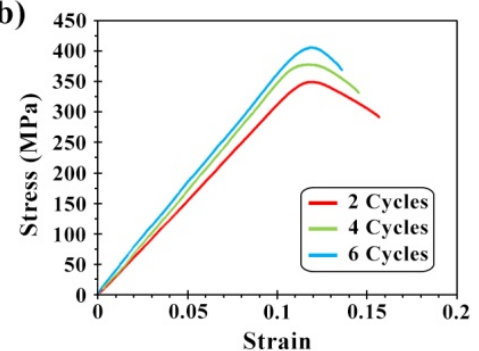

Figure 4. Stress-Strain curves of the (a) RTF and (b) CF samples

a)

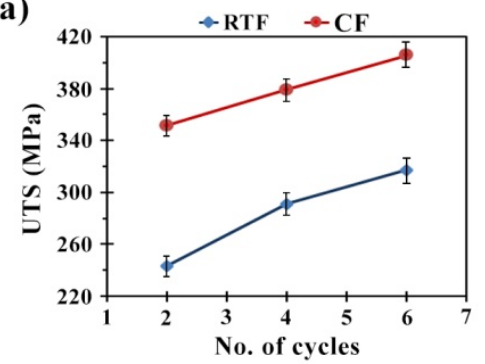

b)

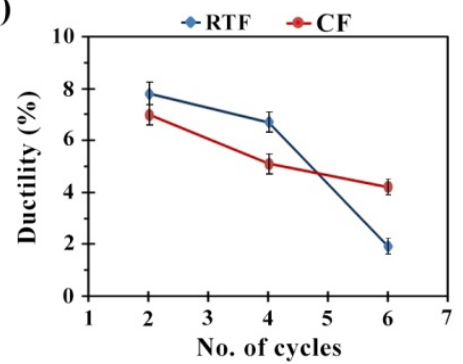

Figure 5. (a) UTS and (b) elongation of the RTF and CF samples vs. number of forging cycles

In comparison with the RTF results, increasing the cycle number plays less significant role in enhancing the UTS of the CF alloys (Figure 4b and Figure 5a), such that only 15\% UTS improvement was observed with proceeding forging from 2 to 6 cycles. This value is about half of the amount of improvement that has been observed in the RTF samples. Regarding this tensile behavior, it can be inferred that the significant portion of the possible grain refinement in the forging process in cryogenic condition was done with only 2 cycles due to the applied intense plastic deformation as well as suppression of the dynamic recovery mechanism. As expected, the hardness measurements in Tables 2 also follow the same trends as the UTS results. In fact, high volume of accumulated grain boundaries in the deformed materials acts as barriers for the movement of dislocations.

Although no notable changes in the mechanical properties of the $\mathrm{CF}$ samples were observed by increasing the number of cycles for the MAF process, their tensile profiles indicate significantly higher strength and toughness than those of the RTF samples. Cryogenic processing offers $44 \%$ more UTS in 2 forging cycles when compared with processing at the ambient temperature. Thus, this process leads to save energy and time. In Cryoforging, the strain hardening is preserved up to the extent to which forging is accomplished. It can be inferred that cryogenic environment ceases dislocation annihilation and consequent softening which is unavoidable during the strain hardening at room temperature. In other words, the effective suppression of cross slip as well as climb of dislocations within cryoforging results in high dislocation density which is not the case for conventional ambient forging. Lee et al. (Lee, Shin, Park, \& Nam, 2004) observed the increase in the UTS from 315 to $522 \mathrm{MPa}$ as a result of cryorolling of 5083 aluminum alloy whereas the UTS increased to $490 \mathrm{MPa}$ after room temperature rolling with the same reduction. In fact, the formation of the UFG microstructure with highly refined grains in the CF materials imparts high mechanical properties as expected and consistent with the Hall-Patch relation (Rao, Singh, Brokmeier, \& Jayaganthan, 2015; Rao et al., 2014).

Table 2. The derived mechanical properties from stress-strain graphs plus hardness

\begin{tabular}{llllll}
\hline Materials & No. of Cycles & Ultimate Tensile Strength (MPa) & Ductility (\%) & ${\text { Toughness }\left(\mathrm{MJm}^{-3}\right)}_{\text {Hardness }(\mathrm{Hv})}$ \\
\hline \multirow{2}{*}{ RTF-AA2219 } & 2 & 243 & 7.8 & 23.12 & 93 \\
& 4 & 291 & 6.7 & 21.93 & 124 \\
& 6 & 317 & 1.9 & 10.98 & 138 \\
CF-AA2219 & 2 & 351 & 7.0 & 36.68 & 161 \\
& 6 & 379 & 5.1 & 35.94 & 175 \\
\end{tabular}


The ultimate strength of aluminum alloys is typically enhanced in the SPD processes at the expense of the ductility (Hung et al., 2005). As can be observed in Figure 5b, cryogenic forging does not only affect significantly the UTS of the CF alloys, but it also increases the elongation of the materials in comparison with the RTF samples after 6 cycles of forging. Ductility is greatly associated with the structural homogeneity in the processed materials. The cryogenic process provides homogeneous grain microstructure with low aspect ratio as a result of the multi axial process associated with recrystallization suppression and intense grain refinement. Nonequilibrium grain boundaries in the UFG materials have also been identified as an effective mechanism to increase ductility (Valiev, Alexandrov, Zhu, \& Lowe, 2002). Such boundaries provide a high density of dislocations for slip which enables grains to slide or rotate during deformation. The decrease in the failure elongation of both the RTF and the CF samples at higher number of forging cycles is another notable point in the strain-strain curves. The lower ductility of the samples processed with higher forging cycles can be mainly attributed to much more limited strain-hardening, which is the result of their inability to pile up dislocations due to the ultrafine grain size and the dislocations saturation (Gong et al., 2013). However, the elongation of the RTF samples was decreased at a faster rate than that of the CF samples indicating a greater grain refinement in the RTF materials between 2 and 6 cycles.

In addition, low strain-hardening was recognized in the stress-strain curves of the tested materials especially in the CF samples. The density of dislocations in nanocrystalline/ultrafine grained materials saturates due to dynamic recovery and annihilation of dislocations into the grain boundaries during the mechanical tests. Since the number of dislocations at the pile-up is significantly reduced, the concept of a pile-up cannot explain the plastic flow (Meyers, Mishra, \& Benson, 2006). The absence of strain hardening resulted in localized deformation leading to low ductility in the RTF alloys and failure in the CF materials.

Using integration method to obtain the area under the true stress-strain curves in Figure 4, the toughness per unit volume of the RTF and the CF specimens was calculated for various cycle numbers. As seen in Table 2, higher amount of energy has been absorbed in the CF samples during the tensile test than the RFT samples. The absorption of the higher portion of the deformation energy indicates higher amount of toughness in the fabricated UFG material. The toughness enhancement in the CF specimens can be ascribed to the refinement of the aluminum grains with consequent high density of dislocations and grain boundary sliding in the UFG structure (Das, Jayaganthan, \& Singh, 2011).

\subsection{Fractography Analysis}

A study on the deformation behavior of the materials is only valid if the failure mechanisms have been well identified by microstructural analysis of the fractured surfaces and failure modes. Figure 6 shows the fractographs of the materials subjected to tensile deformation. Various forging cycles from 2 to 6 were evaluated as a key variable in the two specific RTF and CF processed materials. These surfaces are usually characterized such that the ductile zones are identified by the size and the depth of micro-dimples and the brittle zone has a mere polished morphology. Figure 6a,c,e exhibit the fractured surface of the CF samples processed via 6,4 and 2 forging cycles respectively.

Dimples observed on the fracture surfaces nucleated from the $\mathrm{Al}_{2} \mathrm{Cu}$ second phase particles. As both small micro-dimples and polished morphology are clearly observed, a combination of mostly ductile domain (marked with circles) and small brittle fractured areas (showed with arrows) can be inferred in all the fractured surfaces of the CF alloys. Thus, a typical ductile failure is presented in this material. These were found to be almost similar in terms of the cycle's variation. This similarity is also in consistence with their mechanical behavior shown in the stress-strain profiles (Figure 4). However, a slight ductility reduction is observable with extending the forging process since the dimples became shallower and less in numbers. It can be concluded that a slight mechanical enhancement can be achieved in a considerable amount by increasing the forging cycles in the CF samples after 2 cycles with the associated cost of ductility. 


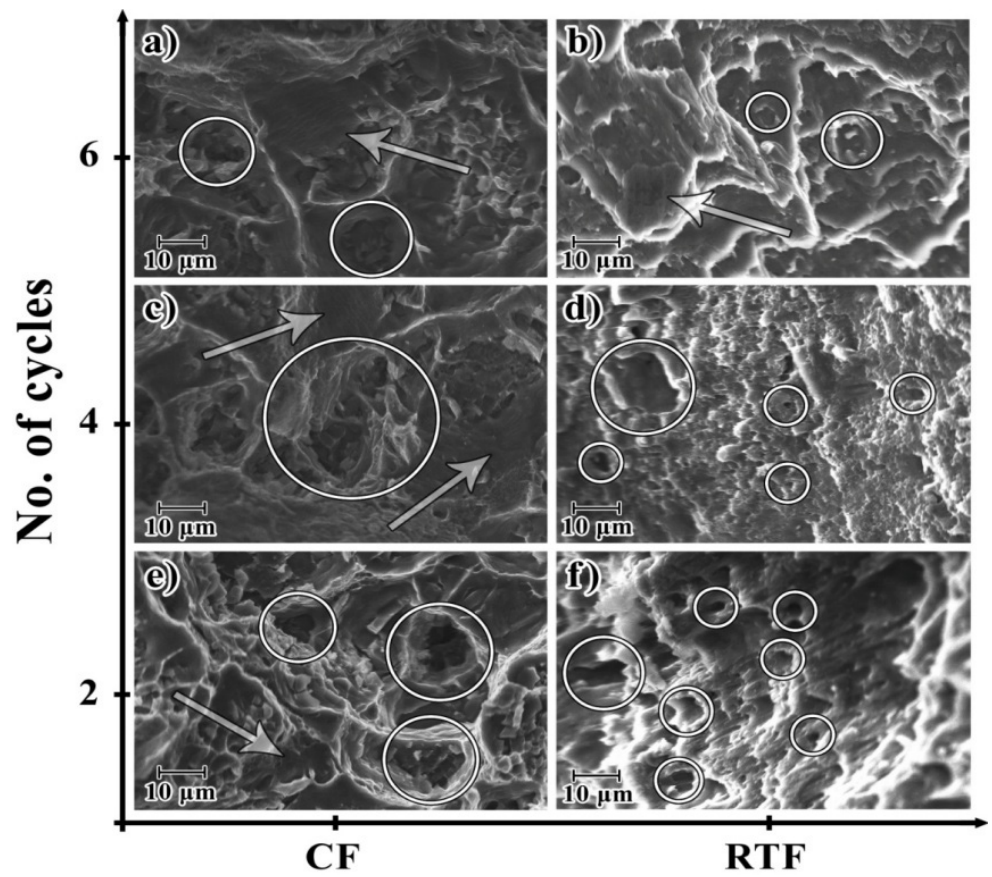

Figure 6. SEM fractographs after tensile test for (a) CF-6 Cycles, (b) RTF-6 Cycles, (c) CF-4 Cycles, (d) RTF-4 Cycles, (e) CF-2 Cycles and (f) RTF-2 Cycles samples

On the other hand, a brittle fractured surface can be observed in the RTF sample up to 6 cycles (Figure $6 \mathrm{~b}$ ) illustrated with large zone with polished morphology. By stepping on the next cycles (Figure 6b,d), the number of deep dimples decreased due to ductility reduction which can be a result of increasing internal strain which keeps inducing intense plastic deformation in the RTF samples. In addition, the finer dimples represent reduced ductility in RTF alloys as compared to the CF samples with coarser and deeper dimples. Therefore, forging at cryogenic temperature improves the ductility of the materials especially in 6 cycle forged samples as it was also observed from the stress-strain plots.

\section{Conclusions}

In this research, AA2219 was processed by multi axial forging (MAF) at ambient and cryogenic temperatures. Then, the microstructure and mechanical behavior of the samples were studied to evaluate the effects of the cryogenic forging on the subgrains dimensions, grain boundaries interactions and the mechanical properties of the tested material. Moreover, fractography analysis was carried out to realize failure mechanisms. The conclusions that can be drawn from this study are summarized as follows:

1) A more effective SPD during the cryogenic MAF was observed in the XRD pattern and the TEM micrographs in the initial microstructure characterization than the same process at room temperature. MAF at cryogenic temperature resulted in crystallite size reduction to nanoscales, heavily deformed grains associated with high density of dislocations and second phase breakage to finer particles which are the crucial factors to increase the mechanical properties of the materials. This can be due to the induced intense plastic deformation as well as the suppression of the dynamic recovery and recrystallization mechanisms.

2) The fractography analysis and tensile tests results indicated that the cryogenic forging does not only enhance significantly the mechanical strength and toughness of the alloys but it also improves the ductility of the materials in comparison with conventional forging. Furthermore, a slight increase in the tensile strength and a ductility reduction resulted at higher cycles of forging due to less ability to accumulate dislocations. However, the elongation of the RTF samples was decreased at a faster rate than that of CF materials especially between 4 and 6 cycles.

\section{Acknowledgement}

Financial support for this work was provided through contract \# W911NF-15-1-0457 under the direct supervision of Patricia Huff (HBCU/MI Program Manager, ARO). 


\section{Conflict of interests}

The authors declare that there is no conflict of interests regarding the publication of this paper.

\section{References}

Asm. (1990). Asm Handbook: Properties and Selection : Nonferrous Alloys and Special-Purpose Materials. Asm Handbook, 2(10). Materials Park, Ohio: ASM International.

Azadmanjiri, J., Berndt, C. C., Kapoor, A., \& Wen, C. (2015). Development of Surface Nano-Crystallization in Alloys by Surface Mechanical Attrition Treatment (SMAT). Critical Reviews in Solid State and Materials Sciences, 40(3), 164-181.

Chen, Y. J., Roven, H. J., Gireesh, S. S., Skaret, P. C., \& Hjelen, J. (2011). Quantitative study of grain refinement in Al-Mg alloy processed by equal channel angular pressing at cryogenic temperature. Materials Letters, 65(23), 3472-3475.

Cherukuri, B., \& Srinivasan, R. (2006). Properties of AA6061 Processed by Multi-Axial Compressions/Forging (MAC/F). Materials and Manufacturing Processes, 21(5), 519-525.

Das, P., Jayaganthan, R., \& Singh, I. V. (2011). Tensile and impact-toughness behaviour of cryorolled A1 7075 alloy. Materials \& Design, 32(3), 1298-1305.

Elgallad, E. M., Zhang, Z., \& Chen, X. G. (2015). Effect of two-step aging on the mechanical properties of AA2219 DC cast alloy. Materials Science and Engineering: A, 625, 213-220.

Hansen, N. (2004). Hall-Petch relation and boundary strengthening. Scripta Materialia, 51(8), 801-806.

Hung, P. C., Sun, P. L., Yu, C. Y., Kao, P. W., \& Chang, C. P. (2005). Inhomogeneous tensile deformation in ultrafine-grained aluminum. Scripta Materialia, 53(6), 647-652.

Ivanov, K. V., \& Naydenkin, E. V. (2012). Grain boundary sliding in ultrafine grained aluminum under tension at room temperature. Scripta Materialia, 66(8), 511-514.

Kumar Singh, A., Ghosh, S., \& Mula, S. (2016). Simultaneous improvement of strength, ductility and corrosion resistance of A12024 alloy processed by cryoforging followed by ageing. Materials Science and Engineering: A, 651, 774-785.

Lee, Y. B., Shin, D. H., Park, K. T., \& Nam, W. J. (2004). Effect of annealing temperature on microstructures and mechanical properties of a $5083 \mathrm{Al}$ alloy deformed at cryogenic temperature. Scripta Materialia, 51(4), 355-359.

Magalhães, D. C. C., Kliauga, A. M., Ferrante, M., \& Sordi, V. L. (2017). Plastic deformation of FCC alloys at cryogenic temperature: The effect of stacking-fault energy on microstructure and tensile behaviour. Journal of Materials Science, 52(12), 7466-7478.

Meyers, M. A., Mishra, A., \& Benson, D. J. (2006). Mechanical properties of nanocrystalline materials. Progress in Materials Science, 51(4), 427-556.

Nejadseyfi, O., Shokuhfar, A., Azimi, A., \& Shamsborhan, M. (2015). Improving homogeneity of ultrafine-grained/nanostructured materials produced by ECAP using a bevel-edge punch. Journal of Materials Science, 50(3), 1513-1522.

Nejadseyfi, O., Shokuhfar, A., Dabiri, A., \& Azimi, A. (2015). Combining equal-channel angular pressing and heat treatment to obtain enhanced corrosion resistance in 6061 aluminum alloy. Journal of Alloys and Compounds, 648, 912-918.

Owolabi, G. M., Bolling, D. T., Odeshi, A. G., Whitworth, H. A., Yilmaz, N., \& Zeytinci, A. (2017). The Effects of Specimen Geometry on the Plastic Deformation of AA 2219-T8 Aluminum Alloy Under Dynamic Impact Loading. Journal of Materials Engineering and Performance, 26(12), 5837-5846.

Panigrahi, S. K., Jayaganthan, R., \& Chawla, V. (2008). Effect of cryorolling on microstructure of Al-Mg-Si alloy. Materials Letters, 62(17), 2626-2629.

Rao, P. N., Singh, D., \& Jayaganthan, R. (2014). Mechanical properties and microstructural evolution of Al 6061 alloy processed by multidirectional forging at liquid nitrogen temperature. Materials \& Design (1980-2015), 56, 97-104.

Rao, P. N., Singh, D., Brokmeier, H. G., \& Jayaganthan, R. (2015). Effect of ageing on tensile behavior of ultrafine grained Al 6061 alloy. Materials Science and Engineering: A, 641, 391-401. 
Sitdikov, O., Sakai, T., Goloborodko, A., \& Miura, H. (2004). Grain fragmentation in a coarse-grained $7475 \mathrm{Al}$ alloy during hot deformation. Scripta Materialia, 51(2), 175-179.

Tan, E., Kibar, A. A., \& Gür, C. H. (2011). Mechanical and microstructural characterization of 6061 aluminum alloy strips severely deformed by Dissimilar Channel Angular Pressing. Materials Characterization, 62(4), 391-397.

Valiev, R. Z., Alexandrov, I. V., Zhu, Y. T., \& Lowe, T. C. (2002). Paradox of Strength and Ductility in Metals Processed Bysevere Plastic Deformation. Journal of Materials Research, 17(1), 5-8.

Valiev, R. Z., Islamgaliev, R. K., \& Alexandrov, I. V. (2000). Bulk nanostructured materials from severe plastic deformation. Progress in Materials Science, 45(2), 103-189.

Xu, S., Zhao, G., Wu, X., Ma, X., \& Guan, Y. (2007). Three-dimensional numerical analysis and experimental investigation of grain refinement in multi-pass equal channel angular pressing for round-workpieces. Frontiers of Materials Science in China, 1(2), 187-196.

\section{Copyrights}

Copyright for this article is retained by the author(s), with first publication rights granted to the journal.

This is an open-access article distributed under the terms and conditions of the Creative Commons Attribution license (http://creativecommons.org/licenses/by/4.0/). 